\title{
ON GENERALIZED k-FRACTIONAL DERIVATIVE OPERATOR
}

\author{
GAUHAR RAHMAN, KOTTAKKARAN SOOPPY NISAR*, SHAHID MUBEEN
}

AbSTRACT. The main objective of this paper is to introduce $\mathrm{k}$-fractional derivative operator by using the definition of $\mathrm{k}$-beta function. We establish some results related to the newly defined fractional operator such as Mellin transform and relations to khypergeometric and $\mathrm{k}$-Appell's functions. Also, we investigate the $\mathrm{k}$-fractional derivative of k-Mittag-Leffler and Wright hypergeometric functions.

\section{INTRODUCTION}

The classical beta function

$$
\beta\left(\delta_{1}, \delta_{2}\right)=\int_{0}^{\infty} t^{\delta_{1}-1}(1-t)^{\delta_{2}-1} d t,\left(\Re\left(\delta_{1}\right)>0, \Re\left(\delta_{2}\right)>0\right)
$$

and its relation with well known gamma function is given by

$$
\beta\left(\delta_{1}, \delta_{2}\right)=\frac{\Gamma\left(\delta_{1}\right) \Gamma\left(\delta_{2}\right)}{\Gamma\left(\delta_{1}+\delta_{2}\right)}, \Re\left(\delta_{1}\right)>0, \Re\left(\delta_{2}\right)>0 .
$$

The Gauss hypergeometric, confluent hypergeometric and Appell's functions which are respectively defined by(see [14])

$$
\begin{array}{r}
{ }_{2} F_{1}\left(\delta_{1}, \delta_{2} ; \delta_{3} ; z\right)=\sum_{n=0}^{\infty} \frac{\left(\delta_{1}\right)_{n}\left(\delta_{2}\right)_{n}}{\left(\delta_{3}\right)_{n}} \frac{z^{n}}{n !},(|z|<1), \\
\left(\delta_{1}, \delta_{2}, \delta_{3} \in \mathbb{C} \text { and } \delta_{3} \neq 0,-1,-2,-3, \cdots\right),
\end{array}
$$

and

$$
\begin{aligned}
& { }_{1} \Phi_{1}\left(\delta_{2} ; \delta_{3} ; z\right)=\sum_{n=0}^{\infty} \frac{\left(\delta_{2}\right)_{n}}{\left(\delta_{3}\right)_{n}} \frac{z^{n}}{n !},(|z|<1), \\
& \quad\left(\delta_{2}, \delta_{3} \in \mathbb{C} \text { and } \delta_{3} \neq 0,-1,-2,-3, \cdots\right), \text { respectively. }
\end{aligned}
$$

The Appell's series or bivariate hypergeometric series is defined by

$$
F_{1}\left(\delta_{1}, \delta_{2}, \delta_{3} ; \delta_{4} ; x, y\right)=\sum_{m, n=0}^{\infty} \frac{\left(\delta_{1}\right)_{m+n}\left(\delta_{2}\right)_{m}\left(\delta_{3}\right)_{n} x^{m} y^{n}}{\left(\delta_{4}\right)_{m+n} m ! n !}
$$

2010 Mathematics Subject Classification. 33B15, 33C15, 33C05, 33C20, 33C65, 33E12, $26 \mathrm{~A} 33$.

$K e y$ words and phrases. Beta function, $k$-beta function, Hypergeometric function, $k$-hypergeometric function, Mellin transform, fractional derivative, Appell's function, $k$-Mittag-Leffler function .

${ }^{*}$ Corresponding author. 
for all $\delta_{1}, \delta_{2}, \delta_{3}, \delta_{4} \in \mathbb{C}, \delta_{4} \neq 0,-1,-2,-3, \cdots, \quad|x|,|y|<1<1$.

The integral representation of hypergeometric, confluent hypergeometric and Appell's functions are respectively defined by

and

$$
\begin{gathered}
{ }_{2} F_{1}\left(\delta_{1}, \delta_{2} ; \delta_{3} ; z\right)=\frac{\Gamma\left(\delta_{3}\right)}{\Gamma\left(\delta_{2}\right) \Gamma\left(\delta_{3}-\delta_{2}\right)} \int_{0}^{1} t^{\delta_{2}-1}(1-t)^{\delta_{3}-\delta_{2}-1}(1-z t)^{-\delta_{1}} d t, \\
\left(\Re\left(\delta_{3}\right)>\Re\left(\delta_{2}\right)>0,|\arg (1-z)|<\pi\right),
\end{gathered}
$$

$$
\begin{aligned}
& { }_{1} \Phi_{1}\left(\delta_{2} ; \delta_{3} ; z\right)=\frac{\Gamma\left(\delta_{3}\right)}{\Gamma\left(\delta_{2}\right) \Gamma\left(\delta_{3}-\delta_{2}\right)} \int_{0}^{1} t^{\delta_{2}-1}(1-t)^{\delta_{3}-\delta_{2}-1} e^{z t} d t, \\
& \left(\Re\left(\delta_{3}\right)>\Re\left(\delta_{2}\right)>0\right) . \\
& F_{1}\left(\delta_{1}, \delta_{2}, \delta_{3} ; \delta_{4} ; x, y\right) \\
& =\frac{\Gamma\left(\delta_{4}\right)}{\Gamma\left(\delta_{1}\right) \Gamma\left(\delta_{4}-\delta_{1}\right)} \int_{0}^{1} t^{\delta_{1}-1}(1-t)^{\delta_{4}-\delta_{1}-1}(1-x t)^{-\delta_{2}}(1-y t)^{-\delta_{3}} d t .
\end{aligned}
$$

The k-gamma function, k-beta function and the k-Pochhammer symbol introduced and studied by Diaz and Pariguan [1]. The integral representation of k-gamma function and k-beta function respectively given by

$$
\begin{aligned}
& \Gamma_{\mathrm{k}}(z)=\mathrm{k}^{\frac{z}{\mathrm{k}}-1} \Gamma\left(\frac{z}{\mathrm{k}}\right)=\int_{0}^{\infty} t^{z-1} e^{-\frac{z^{\mathrm{k}}}{\mathrm{k}}} d t, \quad \Re(z)>0, \mathrm{k}>0 \\
& B_{\mathrm{k}}(x, y)=\frac{1}{\mathrm{k}} \int_{0}^{1} t^{\frac{x}{\mathrm{k}}-1}(1-t)^{\frac{y}{k}-1} d t, \quad \Re(x)>0, \Re(y)>0 .
\end{aligned}
$$

Here, we recall the following relations (see [1]).

$$
\begin{gathered}
B_{\mathrm{k}}(x, y)=\frac{\Gamma_{\mathrm{k}}(x) \Gamma_{\mathrm{k}}(y)}{\Gamma_{\mathrm{k}}(x+y)}, \\
(z)_{n, \mathrm{k}}=\frac{\Gamma_{\mathrm{k}}(z+n \mathrm{k})}{\Gamma_{\mathrm{k}}(z)},
\end{gathered}
$$

where $(z)_{n, \mathrm{k}}=(z)(z+\mathrm{k})(z+2 \mathrm{k}) \cdots(z+(n-1) \mathrm{k}) ; \quad(z)_{0, \mathrm{k}}=1$ and $\mathrm{k}>0$ and

$$
\sum_{n=0}^{\infty}(\alpha)_{n, \mathrm{k}} \frac{z^{n}}{n !}=(1-\mathrm{k} z)^{\frac{-\alpha}{k}} .
$$

These studies were followed by Mansour [6], Kokologiannaki [3], Krasniqi [4] and Merovci [7]. In 2012, Mubeen and Habibullah [8] defined the k-hypergeometric function as

$$
{ }_{2} F_{1, \mathrm{k}}\left(\delta_{1}, \delta_{2} ; \delta_{3} ; z\right)=\sum_{n=0}^{\infty} \frac{\left(\delta_{1}\right)_{n, \mathrm{k}}\left(\delta_{2}\right)_{n, \mathrm{k}}}{\left(\delta_{3}\right)_{n, \mathrm{k}}} \frac{z^{n}}{n !},
$$


where $\delta_{1}, \delta_{2}, \delta_{3} \in \mathbb{C}$ and $\delta_{3} \neq 0,-1,-2, \cdots$ and its integral representation is given by

$$
{ }_{2} F_{1, \mathrm{k}}\left(\delta_{1}, \delta_{2} ; \delta_{3} ; z\right)=\frac{1}{\mathrm{k} \beta_{\mathrm{k}}\left(\delta_{2}, \delta_{3}-\delta_{2}\right)} \int_{0}^{1} t^{\frac{\delta_{2}}{\mathrm{k}}-1}(1-t)^{\frac{\delta_{3}-\delta_{2}}{\mathrm{k}}-1}(1-\mathrm{k} t z)^{-\frac{\delta_{1}}{\mathrm{k}}} d t .
$$

The k-Riemann-Liouville (R-L) fractional integral using $\mathrm{k}$-gamma function introduced in $[9]$ :

$$
\left(I_{\mathrm{k}}^{\alpha} f(t)\right)(x)=\frac{1}{\mathrm{k} \Gamma_{\mathrm{k}}(\alpha)} \int_{0}^{x} f(t)(x-t)^{\frac{\alpha}{\mathrm{k}}-1} d t, \mathrm{k}, \alpha \in \mathbb{R}^{+} .
$$

The solution of some integral equations involving confluent $\mathrm{k}$-hypergeometric functions and $\mathrm{k}$-analogue of Kummer's first formula are given in $[12,13]$. While the $\mathrm{k}$-hypergeometric and confluent $\mathrm{k}$-hypergeometric differential equations are introduced in [10].

In 2015, Mubeen et al. [11] introduced k-Appell hypergeometric function as

$$
F_{1, \mathrm{k}}\left(\delta_{1}, \delta_{2}, \delta_{3} ; \delta_{4} ; z_{1}, z_{2}\right)=\sum_{m, n=0}^{\infty} \frac{\left(\delta_{1}\right)_{m+n, \mathrm{k}}\left(\delta_{2}\right)_{m, \mathrm{k}}\left(\delta_{3}\right)_{m, \mathrm{k}}}{\left(\delta_{4}\right)_{m+n, \mathrm{k}}} \frac{z_{1}^{m} z_{2}^{n}}{m ! n !}
$$

for all $\delta_{1}, \delta_{2}, \delta_{3}, \delta_{4} \in \mathbb{C}, \delta_{4} \neq 0,-1,-2,-3, \cdots, \quad \max \left\{\left|z_{1}\right|,\left|z_{2}\right|\right\}<\frac{1}{\mathrm{k}}$ and $\mathrm{k}>0$.

Also, they define its integral representation as

$$
\begin{aligned}
F_{1, \mathrm{k}}\left(\delta_{1}, \delta_{2}, \delta_{3} ; \delta_{4} ; z_{1}, z_{2}\right) & =\frac{1}{\mathrm{k} \beta\left(\delta_{1}, \delta_{4}-\delta_{1}\right)} \\
& \times \int_{0}^{1} t^{\frac{\delta_{1}}{\mathrm{k}}-1}(1-t)^{\frac{\delta_{4}-\delta_{1}}{\mathrm{k}}-1}\left(1-\mathrm{k} z_{1} t\right)^{-\frac{\delta_{2}}{\mathrm{k}}}\left(1-\mathrm{k} z_{2} t\right)^{-\frac{\delta_{3}}{\mathrm{k}}} d t
\end{aligned}
$$

\section{EXTENSION OF FRACTIONAL DERIVATIVE OPERATOR}

In this section, we recall the definition of following fractional derivatives and give a new extension called Riemann-Liouville $\mathrm{k}$-fractional derivative.

Definition 2.1. The well-known $R$ - $L$ fractional derivative of order $\mu$ is defined by

$$
\mathfrak{D}_{x}^{\mu}\{f(x)\}=\frac{1}{\Gamma(-\mu)} \int_{0}^{x} f(t)(x-t)^{-\mu-1} d t, \Re(\mu)>0 .
$$

For the case $m-1<\Re(\mu)<m$ where $m=1,2, \cdots$, it follows

$$
\begin{aligned}
\mathfrak{D}_{x}^{\mu}\{f(x)\} & =\frac{d^{m}}{d x^{m}} \mathfrak{D}_{x}^{\mu-m}\{f(x)\} \\
& =\frac{d^{m}}{d x^{m}}\left\{\frac{1}{\Gamma(-\mu+m)} \int_{0}^{x} f(t)(x-t)^{-\mu+m-1} d t\right\}, \Re(\mu)>0 .
\end{aligned}
$$

In the following, we define Riemann-Liouville $\mathrm{k}$-fractional derivative of order $\mu$ as

\section{Definition 2.2.}

$$
{ }_{\mathrm{k}} \mathfrak{D}_{x}^{\mu}\{f(x)\}=\frac{1}{\mathrm{k} \Gamma_{\mathrm{k}}(-\mu)} \int_{0}^{x} f(t)(x-t)^{-\frac{\mu}{\mathrm{k}}-1} d t, \Re(\mu)>0, \mathrm{k} \in \mathbb{R}^{+} .
$$

For the case $m-1<\Re(\mu)<m$ where $m=1,2, \cdots$, it follows

$$
\mathrm{k}_{x}^{\mu}\{f(x)\}=\frac{d^{m}}{d x^{m}} \mathfrak{k}_{x} \mathfrak{D}_{x}^{\mu-m \mathrm{k}}\{f(x)\}
$$




$$
=\frac{d^{m}}{d x^{m}}\left\{\frac{1}{\mathrm{k} \Gamma_{\mathrm{k}}(-\mu+m \mathrm{k})} \int_{0}^{x} f(t)(x-t)^{-\frac{\mu}{\mathrm{k}}+m-1} d t\right\}, \Re(\mu)>0 .
$$

Note that for $\mathrm{k}=1$, definition 2.2 reduces to the classical $\mathrm{R}$ - $\mathrm{L}$ fractional derivative operator given in definition 2.1 .

Now, we are ready to prove some theorems by using the new definition 2.2 .

Theorem 2.1. The following formula holds true,

$$
{ }_{\mathrm{k}} \mathfrak{D}_{z}^{\mu}\left\{z^{\frac{\eta}{\mathrm{k}}}\right\}=\frac{z^{\frac{\eta-\mu}{\mathrm{k}}}}{\Gamma_{\mathrm{k}}(-\mu)} \beta_{\mathrm{k}}(\eta+\mathrm{k},-\mu), \Re(\mu)>0 .
$$

Proof. From (2.3), we have

$$
\mathfrak{k}_{z}^{\mu}\left\{z^{\frac{\eta}{\mathrm{k}}}\right\}=\frac{1}{\mathrm{k} \Gamma_{\mathrm{k}}(-\mu)} \int_{0}^{z} t^{\frac{\eta}{\mathrm{k}}}(z-t)^{-\frac{\mu}{\mathrm{k}}-1} d t .
$$

Substituting $t=u z$ in $(2.6)$, we get

$$
\begin{aligned}
& { }_{\mathrm{k}} \mathfrak{D}_{z}^{\mu}\left\{z^{\frac{\eta}{\mathrm{k}}}\right\}=\frac{1}{\mathrm{k} \Gamma_{\mathrm{k}}(-\mu)} \int_{0}^{1}(u z)^{\frac{\eta}{\mathrm{k}}}(z-u z)^{-\frac{\mu}{k}-1} z d u \\
& =\frac{z^{\frac{\eta-\mu}{\mathrm{k}}}}{\mathrm{k} \Gamma_{\mathrm{k}}(-\mu)} \int_{0}^{1} u^{\frac{\eta}{\mathrm{k}}}(1-u)^{-\frac{\mu}{\mathrm{k}}-1} d u \text {. }
\end{aligned}
$$

Applying definition (1.9) to the above equation, we get the desired result.

Theorem 2.2. Let $\Re(\mu)>0$ and suppose that the function $f(z)$ is analytic at the origin with its Maclaurin expansion given by $f(z)=\sum_{n=0}^{\infty} a_{n} z^{n}$ where $|z|<\rho$ for some $\rho \in \mathbb{R}^{+}$. Then

$$
{ }_{\mathrm{k}} \mathfrak{D}_{z}^{\mu}\{f(z)\}=\sum_{n=0}^{\infty} a_{n \mathrm{k}} \mathfrak{D}_{z}^{\mu}\left\{z^{n}\right\} .
$$

Proof. Using the series expansion of the function $f(z)$ in $(2.3)$ gives

$$
{ }_{\mathrm{k}} \mathfrak{D}_{z}^{\mu}\{f(z)\}=\frac{1}{\mathrm{k} \Gamma_{\mathrm{k}}(-\mu)} \int_{0}^{z} \sum_{n=0}^{\infty} a_{n} t^{n}(z-t)^{-\frac{\mu}{\mathrm{k}}-1} d t .
$$

As the series is uniformly convergent on any closed disk centered at the origin with its radius smaller then $\rho$, therefore the series so does on the line segment from 0 to a fixed $z$ for $|z|<\rho$. Thus it guarantee terms by terms integration as follows

$$
\begin{aligned}
\mathrm{k}_{z}^{\mu}\{f(z)\} & =\sum_{n=0}^{\infty} a_{n}\left\{\frac{1}{\mathrm{k} \Gamma_{\mathrm{k}}(-\mu)} \int_{0}^{z} t^{n}(z-t)^{-\frac{\mu}{\mathrm{k}}-1} d t\right. \\
& =\sum_{n=0}^{\infty} a_{n \mathrm{k}} \mathfrak{D}_{z}^{\mu}\left\{z^{n}\right\},
\end{aligned}
$$

which is the required proof. 
Theorem 2.3. The following result holds true:

$$
{ }_{\mathrm{k}} \mathfrak{D}_{z}^{\eta-\mu}\left\{z^{\frac{\eta}{\mathrm{k}}-1}(1-\mathrm{k} z)^{-\frac{\beta}{\mathrm{k}}}\right\}=\frac{\Gamma_{\mathrm{k}}(\eta)}{\Gamma_{\mathrm{k}}(\mu)} z^{\frac{\mu}{\mathrm{k}}-1}{ }_{2} F_{1, \mathrm{k}}(\beta, \eta ; \mu ; z),
$$

where $\Re(\mu)>\Re(\eta)>0$ and $|z|<1$.

Proof. By direct calculation, we have

$$
\begin{aligned}
\mathrm{k}_{\mathfrak{D}^{\prime}}^{\eta-\mu}\left\{z^{\frac{\eta}{\mathrm{k}}-1}(1-\mathrm{k} z)^{-\frac{\beta}{\mathrm{k}}}\right\} & =\frac{1}{\mathrm{k} \Gamma_{\mathrm{k}}(\mu-\eta)} \int_{0}^{z} t^{\frac{\eta}{\mathrm{k}}-1}(1-\mathrm{k} t)^{-\frac{\beta}{\mathrm{k}}}(z-t)^{\frac{\mu-\eta}{\mathrm{k}}-1} d t \\
& =\frac{z^{\frac{\mu-\eta}{\mathrm{k}}-1}}{\mathrm{k} \Gamma_{\mathrm{k}}(\mu-\eta)} \int_{0}^{z} t^{\frac{\eta}{\mathrm{k}}-1}(1-\mathrm{k} t)^{-\frac{\beta}{\mathrm{k}}}\left(1-\frac{t}{z}\right)^{\frac{\mu-\eta}{\mathrm{k}}-1} d t .
\end{aligned}
$$

Substituting $t=z u$ in the above equation, we get

$$
{ }_{\mathrm{k}} \mathfrak{D}_{z}^{\eta-\mu}\left\{z^{\frac{\eta}{\mathrm{k}}-1}(1-\mathrm{k} z)^{-\frac{\beta}{\mathrm{k}}}\right\}=\frac{z^{\frac{\mu}{\mathrm{k}}-1}}{\mathrm{k} \Gamma_{\mathrm{k}}(\mu-\eta)} \int_{0}^{1} u^{\frac{\eta}{\mathrm{k}}-1}(1-\mathrm{k} u z)^{-\frac{\beta}{\mathrm{k}}}(1-u)^{\frac{\mu-\eta}{\mathrm{k}}-1} z d u .
$$

Applying (1.14) and after simplification we get the required proof.

Theorem 2.4. The following result holds true:

$$
\mathrm{k} \mathfrak{D}_{z}^{\eta-\mu}\left\{z^{\frac{\eta}{\mathrm{k}}-1}(1-\mathrm{k} a z)^{-\frac{\alpha}{\mathrm{k}}}(1-\mathrm{k} b z)^{-\frac{\beta}{\mathrm{k}}}\right\}=\frac{\Gamma_{\mathrm{k}}(\eta)}{\Gamma_{\mathrm{k}}(\mu)} z^{\frac{\mu}{\mathrm{k}}-1} F_{1, \mathrm{k}}(\eta, \alpha, \beta ; \mu ; a z, b z),
$$

where $\Re(\mu)>\Re(\eta)>0, \Re(\alpha)>0, \Re(\beta)>0, \max \{|a z|,|b z|\}<\frac{1}{\mathrm{k}}$.

Proof. To prove(2.9), we use the power series expansion

$$
(1-\mathrm{k} a z)^{-\frac{\alpha}{\mathrm{k}}}(1-\mathrm{k} b z)^{-\frac{\beta}{\mathrm{k}}}=\sum_{m=0}^{\infty} \sum_{n=0}^{\infty}(\alpha)_{m, \mathrm{k}}(\beta)_{n, \mathrm{k}} \frac{(a z)^{m}}{m !} \frac{(b z)^{n}}{n !} .
$$

Now, applying Theorem 2.1, we obtain

$$
\begin{aligned}
& \mathrm{k} \mathfrak{D}_{z}^{\eta-\mu}\left\{z^{\frac{\eta}{\mathrm{k}}-1}(1-\mathrm{k} a z)^{-\frac{\alpha}{\mathrm{k}}}(1-\mathrm{k} b z)^{-\frac{\beta}{\mathrm{k}}}\right\} \\
& =\sum_{m=0}^{\infty} \sum_{n=0}^{\infty}(\alpha)_{m, \mathrm{k}}(\beta)_{n, \mathrm{k}} \frac{(a)^{m}}{m !} \frac{(b)^{n}}{n !} \mathfrak{k}_{z}^{\eta-\mu}\left\{z^{\frac{\eta}{\mathrm{k}}+m+n-1}\right\} \\
& =\sum_{m=0}^{\infty} \sum_{n=0}^{\infty}(\alpha)_{m, \mathrm{k}}(\beta)_{n, \mathrm{k}} \frac{(a)^{m}}{m !} \frac{(b)^{n}}{n !} \frac{\beta_{\mathrm{k}}(\eta+m \mathrm{k}+n \mathrm{k}, \mu-\eta)}{\Gamma_{\mathrm{k}}(\mu-\eta)} z^{\frac{\mu}{\mathrm{k}}+m+n-1} \\
& =\sum_{m=0}^{\infty} \sum_{n=0}^{\infty}(\alpha)_{m, k}(\beta)_{n, k} \frac{(a)^{m}}{m !} \frac{(b)^{n}}{n !} \frac{\Gamma_{k}(\eta+m k+n k)}{\Gamma_{k}(\mu+m k+n k)} z^{\frac{\mu}{k}+m+n-1} .
\end{aligned}
$$

In view of (1.16), we get

$$
{ }_{\mathrm{k}} \mathfrak{D}_{z}^{\eta-\mu}\left\{z^{\frac{\eta}{\mathrm{k}}-1}(1-\mathrm{k} a z)^{-\frac{\alpha}{\mathrm{k}}}(1-\mathrm{k} b z)^{-\frac{\beta}{\mathrm{k}}}\right\}=\frac{\Gamma_{\mathrm{k}}(\eta)}{\Gamma_{\mathrm{k}}(\mu)} z^{\frac{\mu}{\mathrm{k}}-1} F_{1, \mathrm{k}}(\eta, \alpha, \beta ; \mu ; a z, b z) .
$$

Theorem 2.5. The following Mellin transform formula holds true:

$$
M\left\{e^{-x}{ }_{\mathrm{k}} \mathfrak{D}_{z}^{\mu}\left(z^{\frac{\eta}{\mathrm{k}}}\right) ; s\right\}=\frac{\Gamma(s)}{\Gamma_{\mathrm{k}}(-\mu)} \beta_{\mathrm{k}}(\eta+\mathrm{k},-\mu) z^{\frac{\eta-\mu}{\mathrm{k}}},
$$

where $\Re(\eta)>-1, \Re(\mu)>0, \Re(s)>0$. 
Proof. Applying the Mellin transform on definition (2.3), we have

$$
\begin{aligned}
& \left.M\left\{e^{-x}{ }_{\mathrm{k}} \mathfrak{D}_{z}^{\mu}\left(z^{\frac{\eta}{\mathrm{k}}}\right) ; s\right\}=\int_{0}^{\infty} x^{s-1} e^{-x}{ }_{\mathrm{k}} \mathfrak{D}_{z}^{\mu}\left(z^{\eta}\right) ; s\right\} d x \\
& =\frac{1}{\mathrm{k} \Gamma_{\mathrm{k}}(-\mu)} \int_{0}^{\infty} x^{s-1} e^{-x}\left\{\int_{0}^{z} t^{\frac{\eta}{\mathrm{k}}}(z-t)^{-\frac{\mu}{\mathrm{k}}-1} d t\right\} d x \\
& =\frac{z^{-\frac{\mu}{\mathrm{k}}-1}}{\mathrm{k} \Gamma_{\mathrm{k}}(-\mu)} \int_{0}^{\infty} x^{s-1} e^{-x}\left\{\int_{0}^{z} t^{\frac{\eta}{\mathrm{k}}}\left(1-\frac{t}{z}\right)^{-\frac{\mu}{\mathrm{k}}-1} d t\right\} d x \\
& =\frac{z^{\frac{\eta-\mu}{\mathrm{k}}}}{\mathrm{k} \Gamma_{\mathrm{k}}(-\mu)} \int_{0}^{\infty} x^{s-1} e^{-x}\left\{\int_{0}^{1} u^{\frac{\eta}{\mathrm{k}}}(1-u)^{-\frac{\mu}{\mathrm{k}}-1} d u\right\} d x
\end{aligned}
$$

Interchanging the order of integrations in above equation, we get

$$
\begin{aligned}
M\left\{e^{-x} \mathfrak{k}_{z}^{\mu}\left(z^{\frac{\eta}{\mathrm{k}}}\right) ; s\right\} & =\frac{z^{\frac{\eta-\mu}{\mathrm{k}}}}{\mathrm{k} \Gamma_{\mathrm{k}}(-\mu)} \int_{0}^{1} u^{\frac{\eta}{\mathrm{k}}}(1-u)^{-\frac{\mu}{\mathrm{k}}-1}\left(\int_{0}^{\infty} x^{s-1} e^{-x} d x\right) d u \\
& =\frac{z^{\frac{\eta-\mu}{\mathrm{k}}}}{\mathrm{k} \Gamma_{\mathrm{k}}(-\mu)} \Gamma(s) \int_{0}^{1} u^{\frac{\eta}{\mathrm{k}}}(1-u)^{-\frac{\mu}{\mathrm{k}}-1} d u \\
& =\frac{\Gamma(s)}{\Gamma_{\mathrm{k}}(-\mu)} \beta_{\mathrm{k}}(\eta+\mathrm{k},-\mu) z^{\frac{\eta-\mu}{\mathrm{k}}}
\end{aligned}
$$

which completes the proof.

Theorem 2.6. The following Mellin transform formula holds true:

$$
M\left\{e^{-x}{ }_{\mathrm{k}} \mathfrak{D}_{z}^{\mu}\left((1-\mathrm{k} z)^{-\frac{\alpha}{\mathrm{k}}}\right) ; s\right\}=\frac{z^{-\frac{\mu}{\mathrm{k}}} \Gamma(s)}{\Gamma_{\mathrm{k}}(-\mu)} \beta_{\mathrm{k}}(\mathrm{k},-\mu)_{2} F_{1, \mathrm{k}}(\alpha, \mathrm{k} ;-\mu+\mathrm{k} ; z),
$$

where $\Re(\alpha)>0, \Re(\mu)<0, \Re(s)>0$, and $|z|<1$.

Proof. Using the power series for $(1-\mathrm{k} z)^{-\frac{\alpha}{\mathrm{k}}}$ and applying Theorem 2.5 with $\eta=n \mathrm{k}$, we can write

$$
\begin{aligned}
M\left\{e^{-x} \mathfrak{k}_{z}^{\mu}\left((1-\mathrm{k} z)^{-\frac{\alpha}{\mathrm{k}}}\right) ; s\right\} & =\sum_{n=0}^{\infty} \frac{(\alpha)_{n, \mathrm{k}}}{n !} M\left\{e^{-x}{ }_{\mathrm{k}} \mathfrak{D}_{z}^{\mu}\left(z^{n}\right) ; s\right\} \\
& =\frac{\Gamma(s)}{\mathrm{k} \Gamma_{\mathrm{k}}(-\mu)} \sum_{n=0}^{\infty} \frac{(\alpha)_{n, \mathrm{k}}}{n !} \beta_{\mathrm{k}}(n \mathrm{k}+\mathrm{k},-\mu) z^{n-\frac{\mu}{\mathrm{k}}} \\
& =\frac{\Gamma(s) z^{-\frac{\mu}{\mathrm{k}}}}{\Gamma_{\mathrm{k}}(-\mu)} \sum_{n=0}^{\infty} \beta_{\mathrm{k}}(n \mathrm{k}+\mathrm{k},-\mu) \frac{(\alpha)_{n, \mathrm{k}} z^{n}}{n !} \\
& =\Gamma(s) z^{-\frac{\mu}{k}} \sum_{n=0}^{\infty} \frac{\Gamma_{k}(k+n k)}{\Gamma_{k}(-\mu+k+n k)} \frac{(\alpha)_{n, k} z^{n}}{n !} \\
& =\frac{\Gamma(s)}{\Gamma_{k}(-\mu+k)} z^{-\frac{\mu}{k}} \sum_{n=0}^{\infty} \frac{(k)_{n, k}}{(-\mu+k)_{n, k}} \frac{(\alpha)_{n, k} z^{n}}{n !} \\
& =\frac{\Gamma(s) z^{-\frac{\mu}{\mathrm{k}}}}{\Gamma_{\mathrm{k}}(-\mu)} \beta_{\mathrm{k}}(\mathrm{k},-\mu)_{2} F_{1, \mathrm{k}}(\alpha, \mathrm{k} ;-\mu+\mathrm{k} ; z),
\end{aligned}
$$

which is the required proof. 
Theorem 2.7. The following result holds true:

$$
{ }_{\mathrm{k}} \mathfrak{D}_{z}^{\eta-\mu}\left[z^{\frac{\eta}{\mathrm{k}}-1} E_{\mathrm{k}, \gamma, \delta}^{\mu}(z)\right]=\frac{z^{\frac{\mu}{\mathrm{k}}-1}}{\mathrm{k} \Gamma_{\mathrm{k}}(\mu-\eta)} \sum_{n=0}^{\infty} \frac{(\mu)_{n, \mathrm{k}}}{\Gamma_{\mathrm{k}}(\gamma n+\delta)} \beta_{\mathrm{k}}(\eta+n \mathrm{k}, \mu-\eta) \frac{z^{n}}{n !},
$$

where $\gamma, \delta, \mu \in \mathbb{C}, \Re(p)>0, \Re(q)>0, \Re(\mu)>\Re(\eta)>0, \Re(\lambda)>0, \Re(\rho)>0$ and $E_{\mathrm{k}, \gamma, \delta}^{\mu}(z)$ is $\mathrm{k}$-Mittag-Leffler function (see [2]) defined as:

$$
E_{\mathrm{k}, \gamma, \delta}^{\mu}(z)=\sum_{n=0}^{\infty} \frac{(\mu)_{n, \mathrm{k}}}{\Gamma_{\mathrm{k}}(\gamma n+\delta)} \frac{z^{n}}{n !} .
$$

Proof. Using (2.13), the left-hand side of (2.12) can be written as

$$
{ }_{\mathrm{k}} \mathfrak{D}_{z}^{\eta-\mu}\left[z^{\frac{\eta}{\mathrm{k}}-1} E_{\mathrm{k}, \gamma, \delta}^{\mu}(z)\right]={ }_{\mathrm{k}} \mathfrak{D}_{z}^{\eta-\mu}\left[z^{\frac{\eta}{\mathrm{k}}-1}\left\{\sum_{n=0}^{\infty} \frac{(\mu)_{n, \mathrm{k}}}{\Gamma_{\mathrm{k}}(\gamma n+\delta)} \frac{z^{n}}{n !}\right\}\right] .
$$

By Theorem 2.2, we have

$$
\mathfrak{k}_{z}^{\eta-\mu}\left[z^{\frac{\eta}{\mathrm{k}}-1} E_{\mathrm{k}, \gamma, \delta}^{\mu}(z)\right]=\sum_{n=0}^{\infty} \frac{(\mu)_{n, \mathrm{k}}}{\Gamma_{\mathrm{k}}(\gamma n+\delta)}\left\{\mathrm{k} \mathfrak{D}_{z}^{\mu}\left[z^{\frac{\eta}{\mathrm{k}}+n-1}\right]\right\} .
$$

In view of Theorem 2.1, we get the required proof.

Theorem 2.8. The following result holds true:

$$
\begin{aligned}
& { }_{\mathrm{k}} \mathfrak{D}_{z}^{\eta-\mu}\left\{z^{\frac{\eta}{\mathrm{k}}-1}{ }_{m} \Psi_{n}\left[\begin{array}{c}
\left(\alpha_{i}, A_{i}\right)_{1, m} ; \\
\left(\beta_{j}, B_{j}\right)_{1, n} ;
\end{array}\right]\right\}=\frac{z^{\frac{\mu}{\mathrm{k}}-1}}{\mathrm{k} \Gamma_{\mathrm{k}}(\mu-\eta)} \\
& \times \sum_{n=0}^{\infty} \frac{\prod_{i=1}^{m} \Gamma\left(\alpha_{i}+A_{i} n\right)}{\prod_{j=1}^{n} \Gamma\left(\beta_{j}+B_{j} n\right.} \beta_{\mathrm{k}}(\eta+n \mathrm{k}, \mu-\eta) \frac{z^{n}}{n !},
\end{aligned}
$$

where $\Re(p)>0, \Re(q)>0, \Re(\mu)>\Re(\eta)>0, \Re(\lambda)>0, \Re(\rho)>0$ and ${ }_{m} \Psi_{n}(z)$ is the Fox-Wright function defined by (see [5], pages 56-58)

$$
{ }_{m} \Psi_{n}(z)={ }_{m} \Psi_{n}\left[\begin{array}{c}
\left(\alpha_{i}, A_{i}\right)_{1, m} ; \\
\left(\beta_{j}, B_{j}\right)_{1, n} ;
\end{array}\right]=\sum_{n=0}^{\infty} \frac{\prod_{i=1}^{m} \Gamma\left(\alpha_{i}+A_{i} n\right)}{\prod_{j=1}^{n} \Gamma\left(\beta_{j}+B_{j} n\right.} \frac{z^{n}}{n !} .
$$

Proof. Applying Theorem 2.1 and followed the same procedure used in Theorem 2.7, we get the desired result.

\section{CONCLUding REMARKS}

In this paper, we established $\mathrm{k}$-fractional derivative operator. If letting $\mathrm{k} \rightarrow 1$ then all the results established in this paper will reduce to the results related to the classical Reimann-Liouville fractional derivative operator. 


\section{REFERENCES}

[1] R. Diaz, E. Pariguan, On hypergeometric functions and Pochhammer k-symbol, Divulgaciones Mathematics, 15 (2), (2007), 179-192.

[2] G. A. Dorrego, R. A. Cerutti, The k-Mittag-Leffler function, Int. J. Contemp. Math. Sciences, 7 (15), (2012), 705-716.

[3] C. G. Kokologiannaki, Properties and inequalities of generalized k-gamma, beta and zeta functions, Int. J. Contemp. Math. Sciences, 5 (2010), 653-660.

[4] V. Krasniqi, A limit for the k-gamma and k-beta function, Int. Math. Forum, 5 (2010), 1613-1617.

[5] A. A. Kilbas, H. M. Sarivastava, J. J. Trujillo, Theory and application of fractional differential equation, North-Holland Mathematics Studies, Elsevier Sciences B.V., Amsterdam, (2006).

[6] M. Mansour, Determining the $k$-generalized gamma function $\Gamma_{k}(x)$ by functional equations, International Journal of Contemporary Mathematical Sciences, 4 (21), (2009), 1037-1042.

[7] F. Merovci, Power product inequalities for the $\Gamma_{k}$ function, Int. J. of Math. Analysis, 4 (2010), 1007-1012.

[8] S. Mubeen, $k$-Analogue of Kummer's first formula, Journal of Inequalities and Special Functions, 3, (2012), 41-44.

[9] S. Mubeen, Solution of Some integral equations involving confluent $k$-hypergeometric functions, Applied Mathematics, 4, (2013), 9-11.

[10] S. Mubeen, G. M. Habibullah, An integral representation of k-hypergeometric functions, International Mathematical Forum, 7 (4), (2012), 203-207.

[11] S. Mubeen, G. M. Habibullah, $k$-fractional integrals and application, Int. J. Contemp. Math. Science, 7 (2), (2012), 89-94.

[12] S. Mubeen, S. Iqbal, G. Rahman, Contiguous function relations and an integral representation for Appell $k$-series $F_{1, k}$, International Journal of Mathematical Research, 4 (2), (2015), 53-63.

[13] S. Mubeen, M. Naz, G. Rahman, A note on k-hypergeometric differential equations, Journal of Inequalities and Special Functions, 4 (3), (2013), 38-43.

[14] E. D. Rainville, Special functions, The Macmillan Company, New York, 1960.

Gauhar Rahman: Department of Mathematics, International Islamic University, IsLAMABad, PAKISTAN

E-mail address: gauhar55uom@gmail.com

Kottakkaran Sooppy Nisar: Department of Mathematics, College of Arts and ScienceWadi Aldawaser, 11991, Prince Sattam bin Abdulaziz University,Alkharj, Kingdom of SAUdi ARABia

E-mail address: n.sooppy@psau.edu.sa; ksnisar1@gmail.com 
ON GENERALIZE k-FRACTIONAL DERIVATIVE OPERATOR

Shahid Mubeen: Department of Mathematics, University of Sargodha, Sargodha, PakISTAN

E-mail address: smjhanda@gmail.com 